\title{
Diacronie
}

Studi di Storia Contemporanea

$N^{\circ} 11,3 \mid 2012$

La satira fa storia. Eventi, pratiche, linguaggi

\section{Nota introduttiva n. 11 - ottobre 2012}

\section{Alessandro Cattunar e Alice de Rensis}

\section{(2) OpenEdition}

\section{Journals}

\section{Edizione digitale}

URL: http://journals.openedition.org/diacronie/2620

DOI: 10.4000/diacronie.2620

ISSN: 2038-0925

\section{Editore}

Association culturelle Diacronie

\section{Notizia bibliografica digitale}

Alessandro Cattunar e Alice de Rensis, « Nota introduttiva n. 11 - ottobre 2012 », Diacronie [Online], № 11, 3 | 2012, Messo online il 29 octobre 2012, consultato il 24 septembre 2020. URL : http:// journals.openedition.org/diacronie/2620; DOI : https://doi.org/10.4000/diacronie.2620

Questo documento è stato generato automaticamente il 24 settembre 2020.

Creative Commons License 
Nota introduttiva n. 11 - ottobre 2012

Alessandro Cattunar e Alice de Rensis

Storia e satira. Una relazione complessa 
1 La satira è una pratica discorsiva complessa e multiforme, una forma d'espressione "di confine", a cavallo tra diversi media, linguaggi e obiettivi. Questo carattere si evidenzia a partire dall'incerta definizione del termine che, in prima battuta, può essere ricondotto ai mitici satiri romani (sàtyros) - la cui natura demoniaca e priapesca rimanda alla sfera della lascivia, dell'aggressività e del sovvertimento delle regole - ma anche alla lanx satura, un piatto rituale ricolmo di cibi diversi ${ }^{1}$. Se nel primo caso l'etimologia ci può suggerire un preciso rapporto con la realtà e il potere, vedendo nella satira il simbolo stesso della libertà d'espressione, nel secondo ci si sofferma maggiormente sulle caratteristiche linguistico-formali del genere,

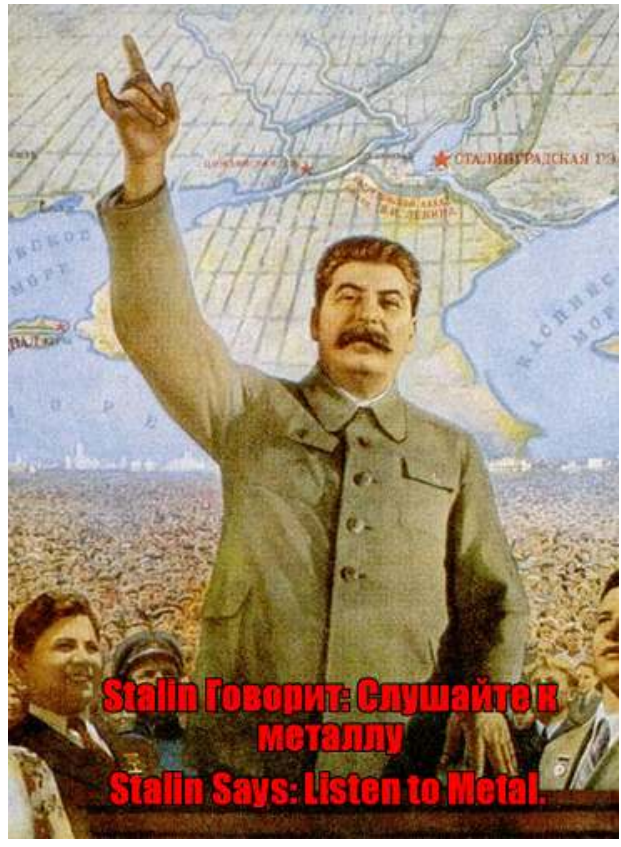
sottolineandone «la mescolanza e la saturazione delle forme, dei generi, degli stili, dei linguaggi marcatamente settoriali, privatistici e spesso osceni, il bricolage parodistico e l'estemporaneità»².

2 Temi, finalità, modi d'espressione, contesti: per comprendere cos'è la satira e come funziona bisogna riflettere congiuntamente su tutte queste dimensioni, intrecciandole ed evidenziando come si influenzino reciprocamente.

3 La volontà di suscitare la risata, nelle produzioni satiriche, si accompagna sempre ad un intento morale, pedagogico oppure pratico: scopo della satira è mettere in luce e deridere $\mathrm{i}$ vizi di singoli individui, di specifici gruppi o dell'intera società, sottolineandone le incongruenze, le contraddizioni e le ipocrisie. Alla pars destruens, quasi sempre in primo piano, in molti casi si affianca un intento più costruttivo, volto a prospettare valori alternativi, nuove visioni del mondo e a proporre "buone pratiche" da seguire o imitare.

4 Per ottenere questi risultati gli autori satirici si servono di media, linguaggi e codici anche molto differenti in cui non sempre è semplice individuare elementi comuni.

5 L'undicesimo numero di Diacronie si propone di studiare questo genere e le sue specificità attraverso i molteplici legami che s'instaurano con l'analisi storica e con le pratiche storiografiche. Non s'intende fare "storia della satira" ma provare, innanzitutto, a riflettere su come le produzioni satiriche di diverse epoche possano essere d'aiuto nello studio e nell'interpretazione degli eventi storici, delle dinamiche sociali e culturali, e nel delineare una storia delle mentalità. Si cercherà poi di analizzare come le produzioni satiriche siano diventate dei veri e propri "attori storici", ritagliandosi un importante ruolo in ambito politico e sociale. Si tratta di un doppio sguardo che pone al centro dell'attenzione soprattutto problematiche di carattere discorsivo, linguistico e metodologico, ma all'interno di un preciso campo di interessi, legato alla ricostruzione e all'interpretazione di fatti, personaggi e dinamiche del passato. 
6 Un primo elemento di riflessione, comune a molti degli autori che hanno contribuito a questo numero, concerne l'individuazione di cosa possa essere considerato satira, quali produzioni culturali e tecniche di scrittura/rappresentazione possano rientrare all'interno di questo genere e quali siano le relazioni con pratiche affini, come la comicità, l'umorismo e l'ironia.

7 Si tratta di distinzioni spesso sottili e i punti di contatto possono sembrare più marcati rispetto alle differenze. A predominare, in tutti questi generi, è "l'effetto" ottenuto, cioè il riso provocato nel pubblico. Più difficile è comprenderne la natura, l'origine e la finalità in quanto non è sufficiente soffermarsi sulle intenzioni degli autori ma bisogna studiare anche la dimensione della ricezione, i saperi, le aspettative e gli umori del pubblico. Infatti, se la satira condivide esplicitamente con il comico la ricerca della risata tramite la messa in ridicolo di fatti o persone, più sfumati appaiono i legami con il sarcasmo - in cui a contare è l'intento beffardo - e con l'ironia, in cui appare centrale il significato volutamente stravolto attribuito alle parole. Anche i confini tra satira e umorismo risultano spesso labili, soprattutto se ci si sofferma sull'esplicito appello che entrambe le pratiche rivolgono all'emotività del destinatario, facendo appello alle pulsioni più basse e agli istinti più naturali.

In epoca contemporanea, con l'allargarsi di queste pratiche ai nuovi mezzi di comunicazione, è diventato ancora più difficile - e non sempre utile, soprattutto per $\mathrm{i}$ nostri fini - mantenere delle rigide distinzioni.

9 Se all'interno degli studi accademici d'area letteraria e artistica la satira è stata per lo più analizzata in quanto genere che, attraverso specifici codici linguistici, mette in risalto e critica aspetti della società, della cultura e della politica contemporanee, col duplice fine di far ridere e di far riflettere, in ambito storiografico sembra predominare una certa disattenzione, se non addirittura diffidenza. Lo mettono chiaramente in evidenza, nelle nostre pagine, Francescomaria Evangelisti e Maria Eugenia Gutiérrez Jiménez - che fanno riferimento alla distrazione di una parte della storiografia verso la cosiddetta "cultura popolare" - e Valerio Zandonà, che riconduce tale atteggiamento proprio alla «natura cangiante e complessa di questa materia».

10 Le analisi proposte da Diacronie cercano di testimoniare questa fluidità definitoria, individuando elementi satirici in diversi tipi di produzioni e all'interno di ambiti estremamente vari: dalla letteratura "alta" di metà Ottocento alle barzellette contemporanee, dagli articoli satirici di un giornale/partito come «L'Uomo qualunque» alle vignette caricaturali, dalla letteratura alla satira su internet.

11 Se abbiamo deciso di affrontare le produzioni satiriche con gli occhi e gli strumenti dello storico, è perché, da un lato, risultano profondamente legate al contesto geografico e cronologico all'interno del quale prendono forma e dall'altro mettono in evidenza la volontà di ottenere un risultato concreto che vada oltre la semplice dimensione del riso o della riflessione arguta. La satira diventa spesso strumento attraverso cui influenzare il pubblico, attaccare gli avversari, promuove il cambiamento: può essere considerata un vero e proprio mezzo d'azione politica e sociale.

Della satira [...] deve essere individuato innanzitutto il fine immediato, da ravvisarsi nella volontà di provocare il sorriso ma [...], mentre nella comicità il riso è fine a se stesso, nella satira esso diviene un mezzo espressivo per raggiungere un fine ulteriore, più o meno serio ${ }^{3}$. 
12 La satira è una forma espressiva strettamente legata all'epoca che la produce, al punto che, se analizzati al di fuori del loro contesto, i testi satirici rischiano di risultare incomprensibili ${ }^{4}$. Al tempo stesso, le figure attaccate dalla satira, siano esse personaggi politici o "tipi" umani, possono essere innalzati ad "archetipi" presenti in tutti i secoli e in tutte le culture.

13 Proprio il rapporto tra satira e contesto storico rappresenta il punto focale di molti dei casi di studio proposti in questa sede. Maurizio Cocco, ad esempio, lo mette bene in evidenza per quanto riguarda gli interventi di Guglielmo Giannini ne «L'Uomo qualunque», ricordando come sia proprio nei «momenti dipiù acceso scontro tra $i$ partiti e le fazioni» $»^{5}$ che si sviluppa la satira più forte e tagliente. Altro elemento su cui soffermare l'attenzione è l'orientamento politico degli autori e la loro capacità di evocare "lo spirito del tempo", in un'ottica che apparirà senza dubbio "partigiana" ma che proprio per questo può fornire molteplici spunti di riflessione al ricercatore attento ai linguaggi della politica. In tal senso, può risultare interessante l'accostamento tra Giannini e Guareschi che, rievocando le parole di Adolfo Chiesa, appaiono «un po' monarchici e un po' anarchici, certo reazionari ma capaci di dialogare con la gente, di far capire ai loro lettori, con decenni di anticipo, tanti malanni della politica, magagne della società, difetti delle istituzioni che solo tanto tempo dopo sarebbero venuti alla luce in tutta la loro evidenza» ${ }^{6}$.

Sempre in quest'ambito di riflessioni, una prospettiva di più lungo respiro viene proposta da Iñigo Fernandez che analizza le barzellette e le battute riferite ai messicani nell'ultimo quarto di secolo evidenziando come queste affondino molto spesso le radici in traumi storici legati ad un passato anche molto lontano ${ }^{7}$.

Nella valutazione dell'efficacia e dell'impatto di un intervento satirico, non ci si può focalizzare unicamente sui contenuti espliciti proposti dai testi ma è opportuno provare a mettere in luce anche le pratiche di socializzazione legate alla fruizione, che risultano spesso determinanti quanto i messaggi stessi, come sottolineano, tra gli altri, Evangelisti e Gutiérrez Jiménez, nella loro analisi della letteratura di cordel nella Spagna di metà Ottocento ${ }^{8}$.

16 L'elemento che maggiormente sembra accomunare i casi di studio presi in esame in questa sede è la volontà degli autori satirici di rappresentare il proprio tempo smascherandone i vizi, il malcostume e gli aspetti grotteschi ma anche proponendo e promuovendo nuovi argomenti di riflessione in grado di promuovere il cambiamento.

Attraverso la rottura delle leggi della logica o la generazione di una collisione tra di esse, si cerca di suscitare indignazione o ilarità nel pubblico, a ciò mossi dal desiderio [...] di provocare la riflessione su aspetti del costume politico, della società, a tal punto radicati nella coscienza sociale da essere accettati, assai spesso, senza alcuno spirito critico'.

Il pubblico è innanzitutto invitato a riflettere su se stesso, sui propri comportamenti e responsabilità, sull'accondiscendenza che spesso dimostra verso atteggiamenti poco virtuosi. Questo approccio moralistico individua poi come bersaglio privilegiato la sfera pubblica e in particolare i rappresentanti dei ceti dominanti, scagliandosi contro «i difetti delle istituzioni e degli individui» ${ }^{10}$. Nelle critiche mosse al mondo e ai rappresentanti della politica l'intento propositivo, volto ad un miglioramento della situazione in essere, lascia spesso spazio ad un più generico atteggiamento di biasimo a tutto campo e di rifiuto radicale di una classe dirigente percepita come incapace, corrotta, in ogni caso ridicola e inadeguata ad affrontare la situazione. Non è un caso - 
e non è solo la volontà di creare un collegamento con la situazione politica attuale -se ricorre spesso, nei saggi di questo numero, l'uso del termine "antipolitica". Ad esempio, "L'Uomo qualunque», soprattutto attraverso la sua rubrica «Le vespe», fece tutto il possibile per delegittimare i rappresentanti delle neonate istituzioni democratiche, che fondavano il proprio riconoscimento nell'esperienza resistenziale, criticando, con aneddoti e battute, il delicato memento di transizione dal fascismo alla vita democratica ${ }^{11}$.

18 La distanza abissale fra "paese reale" e "paese legale" continuamente sottolineata da Giannini nel suo giornale appare quasi del tutto immutata rispetto a quella descritta, in età giolittiana, sulle pagine del «Travaso delle idee» ${ }^{12}$.

19 Com'è noto è soprattutto nei momenti di crisi, nelle fasi di passaggio fra diversi sistemi politico-istituzionali che la satira va a ricoprire un ruolo fondamentale nella circolazione delle idee e nella mobilitazione delle persone, in quanto appare capace di esprimere - in modo sintetico ed estremamente efficace - una sferzante critica verso il potere e, al contempo, una forte carica utopistica e innovatrice. Si tratta di aspetti che Barbara De Poli pone bene in evidenza in riferimento ad alcuni regimi autoritari del Vicino Oriente e del Nord Africa che proprio in questi anni risultano coinvolti in processi di mutamento profondi con la progressiva affermazioni di movimenti che si propongono come democratici. In questi contesti, la satira appare al contempo come uno strumento di opposizione, un mezzo di mobilitazione e una forma privilegiata di libera espressione di speranze e aspettative comuni all'intera società:

l'umorismo che le società arabe affermano quale formula di resistenza, di libertà e di rivendicazione politica sembra, mai come in questa fase della storia, riflettere l'osservazione sull'umorismo di Henri Bergson: "È qualcosa come la logica del sogno, ma d'un sogno che non venga abbandonato al capriccio della fantasia individuale, bensì il sogno sognato dell'intera società" ${ }^{13}$.

20 A rivelarsi particolarmente interessanti, agli occhi dello storico, sono proprio i complessi e multisfaccettati rapporti che si instaurano tra satira e potere. Si tratta di una relazione di vecchia data che, come sottolinea ancora De Poli «trova i suoi interpreti e i suoi censori sin dall'Atene di Aristofane» ${ }^{14}$. Nel caso di regimi autoritari e illiberali - di cui ci parla anche Miguel De Toro Muñoz nel suo saggio sulla satira durante il Terzo Reich ${ }^{15}$ - divengono oggetto d'analisi privilegiata sia i contenuti eversivi, sia i tentativi del potere stesso di far tacere e censurare le poche voci di dissenso che riescono a esprimersi e diffondersi:«il fatto che il Potere, specie se autoritario, debolmente legittimato e deficitario sul piano politico, si preoccupi di oscurare la critica umoristica sempre con costanza e fermezza è di per sé indice del potenziale destabilizzatore di quest'ultima, capace al contempo di denunciare le tare dei governi e di sminuirli ridicolizzandoli» ${ }^{16}$.

21 Molti degli studi proposti in questo numero si soffermano sugli "effetti concreti" che alcuni autori e pubblicazioni hanno avuto all'interno del panorama sociale e politico, diventando dei veri e propri attori sulla scena ideologica e culturale ma anche nelle dinamiche parlamentari e governative in senso stretto. Come ricorda Attilio Brilli, la satira ha contribuito spesso a «trasmettere la storia di una tradizione eversiva, contestataria, tesa a capovolgere [...] strutture gerarchiche cristallizzate e carismatiche figure del potere $»^{17}$.Se la satira militante dell'«Uomo qualunque», nel secondo dopoguerra, ricoprì senza dubbio un importante ruolo nella conquista di trenta seggi all'Assemblea costituente da parte dell'omonimo partito fondato da Giannini ${ }^{18}$, Marco 
Viscardi sottolinea come anche la satira di matrice più prettamente letteraria sia stata utilizzata come arma di battaglia politica nell'Italia di metà Ottocento ${ }^{19}$. Altrettanto stimolanti appaiono, in questa direzione, le analisi di Fernandez sulla valenza acquisita da certe barzellette sui messicani come mezzo di trasgressione contro l'apparato ${ }^{20} \mathrm{e}$ il ruolo ricoperto dalla satira all'interno del Movimento urbano a Valladolid nella Spagna degli anni Settanta ${ }^{21}$.

Se la satira può diventare strumento di lotta politica contro i poteri costituiti, allo stesso modo non si deve dimenticare la forza che può assumere come mezzo di propaganda utilizzato dal potere in modo più o meno esplicito e dichiarato. Basti pensare, per citare un tema già ampiamente sviscerato in altre sedi, agli effetti ottenuti dalla massiccia campagna propagandistica nazista contro il popolo ebraico.

Un altro elemento spesso sottolineato dagli autori di questo numero è l'importanza della satira per lo studio della storia delle mentalità e degli immaginari collettivi. I testi satirici, infatti, non si rivolgono solamente verso l'esterno, avanzando feroci critiche dell' "altro da sé", ma sono in grado di proporre anche uno sguardo, spesso inedito, sulla stessa individualità o collettività che quel testo ha prodotto, sul suo modo di rappresentarsi e autopercepirsi, sulla "configurazione dei ruoli, delle identità e delle coscienze del suo pubblico, così come nella trasformazione degli habitus» ${ }^{22}$.Troviamo quindi un approccio alla satira in quanto "rappresentazione" che scaturisce da una mediazione, più o meno esplicita e consapevole, tra l'autore, il pubblico di riferimento e la società nel suo insieme.

Come ricorda Fernandez, la battuta può acquisire una dimensione antropologica, diventando uno specchio in cui i membri di una società cercano il proprio riflesso ${ }^{23}$.

Strettamente connessi a quest'ambito di discorsi appaiono i complessi legami che intercorrono tra satira e sfera emotiva. Anche in questo caso la relazione è duplice: se da un lato le emozioni - generalmente declinate nelle loro versioni più estreme diventano l'elemento centrale della rappresentazione, dall'altro, vignette, caricature, espressioni verbali sono spesso concepite con il fine esplicito di suscitare reazioni emotive nel pubblico. Si potrebbe dire che, certe volte, la satira parla alla pancia più che alla testa, attraverso l'uso di un linguaggio estremamente colorito e di immagini particolarmente esplicite in cui ad essere colpite sono per lo più la sfera del corpo e della sessualità.

Si tratta di riflessioni che vengono esplicitamente affrontate nel contributo di Dario Pasquini - che sottolinea l'effetto performativo diretto di questo richiamo della satira alla sfera emotiva ${ }^{24}$ - ma anche da Francescomaria Evangelisti e Maria Eugenie Gutiérrez Jiménez, secondo cui la letteratura di cordel si appella all'«appetito popolare per il violento, il truculento e il passionale, sentimenti esclusi dalla cultura ufficiale» ${ }^{25}$. Da questa veloce disanima appare chiara l'importanza di soffermarsi non solo sul "cosa" di ciò viene espresso ma anche sul "come", sui significanti, sugli elementi più prettamente linguistici, provando ad analizzare, ad esempio, i molteplici legami tra la dimensione grafico-lessicale e l'ideologia che l'ha prodotta, analizzando gli specifici codici linguistici, la loro multiformità e le loro contraddizioni, oltre che i legami con altri generi.

Si tratta di un tipo di approfondimento che richiede strumenti specifici, che generalmente non fanno parte della tanto citata "cassetta degli attrezzi" a disposizione dello storico. Gli autori di questo numero, si cimentano con queste difficoltà trovando 
spesso una mediazione tra i limiti derivanti dal leggere queste produzioni «al di fuori del mezzo che le esprime - il linguaggio o la grafica - [...] e il rischio opposto che è quello di studiare la satira come forma linguistica, ignorando la presenza storica che in vario modo l'attraversa e la condiziona» ${ }^{26}$.

La rilevanza degli aspetti linguistici appare in modo estremamente evidente, ancora una volta, negli scritti di Giannini in cui il «linguaggio in sé - forse più del contenuto fungeva da strumento di critica e di opposizione. [...] La satira fu quindi, come ampiamente rivendicato dal giornale, non solo un contenitore ma un contenuto in sé, un modo di differenziarsi, di offrire qualcosa di nuovo e diverso, di codificare un atteggiamento così come l'antipolitica è un modo di fare politica» ${ }^{27}$.

Altrettanto penetrante appare l'analisi linguistica e retorica proposta da Viscardi sui testi satirici di alcuni grandi letterati di metà Ottocento, primi fra tutti quelli di Leopardi, in cui «le forme metriche utilizzate - gli endecasillabi sciolti per la Palinodia, il capitolo in terza rima dei Nuovi Credenti e le ottave del genere eroicomico dei Paralipomeni alla Batriacomiomachia - quasi costringono la tradizione italiana al racconto di un presente degradato e risibile» ${ }^{28}$.

31 Molte volte la satira disvela tutte le sue potenzialità quando fuoriesce dalla limitante bidimensionalità della pagina scritta (o stampata) per assumere nuove forme e significati all'interno della trasmissione orale. Testi satirici come i cordel - "che raccontavano storie adatte a essere lette, recitate e ascoltate da tutti, anche da chi non sapeva leggere ${ }^{29}$ - svelano così il carattere ibrido di molte produzioni umoristiche.

Ma la rilevanza della dimensione non scritta nei meccanismi di diffusione di contenuti satirici traspare soprattutto nel caso delle barzellette che - soprattutto quanto sono rivolte contro regimi totalitari rigidamente censori - trovano modo di diffondersi «solamente tramite l'oralità popolare, sfuggente e imprendibile strumento dell'eversione virtuale - ben prima dell'invenzione di internet $»^{30}$.

Ecco che, in conclusione, ci troviamo nuovamente a sottolineare la natura "di confine" della satira, una natura che rende l'interpretazione storica estremamente complessa perché da un lato non sempre risulta semplice reperire le fonti necessarie (soprattutto se entriamo nell'ambito della trasmissione orale) e dall'altro appare difficoltosa l'analisi della ricezione di testi in cui spesso il significato viene costruito congiuntamente dall'autore e dal fruitore.

Sarebbe stato stimolante - ma è un'impresa che rimandiamo ad un'altra occasione cercare di capire in che modo la satira prodotta oggi ci parli del passato e in che modo gli "autori satirici" assumano, a volte, il ruolo di storici, influenzando le percezioni del pubblico. Sempre più frequentemente vengono pubblicati lavori che si collocano in uno spazio interstiziale estremamente labile tra due pratiche e due linguaggi apparentemente agli antipodi: appunto storiografia e satira. Il comico, il vignettista, il fumettista spesso affrontano in maniera tutt'altro che superficiale tematiche storiche estremamente complesse attraverso linguaggi ibridi. Si pensi, solo per fare un esempio, ai numerosi graphic novel basati su ricerche storiche approfondite che vengono comunicate attraverso un linguaggio che fonde toni saggistici e codici propri della satira, dell'umorismo e della caricatura.

Con questo numero di Diacronie speriamo di essere riusciti a porre sul tavolo almeno una parte delle numerose e complesse problematiche che caratterizzano il rapporto tra satira e storia, proponendo alcuni casi di studio che toccano contesti geografici e 
periodi storici anche molto lontani. Nonostante queste distanze, i saggi presentati rivelano numerosi punti di contatto e possono essere proficuamente collegati e riportati ad una prospettiva d'insieme, tanto che sarebbe stata una forzatura raggrupparli in sezioni distinte.

\section{NOTE}

1. Traggo queste riflessioni da BRILLI, Attilio, Dalla satira alla caricatura. Storia, tecniche e ideologie della rappresentazione, Bari, edizioni Dedalo, 1985, p. 8-9.

2. Ibidem, p. 9.

3. BALESTRA, Luigi, La satira come forma di manifestazione del pensiero. Fondamenti e limiti, Milano, Giuffré 1998, p. 2.

4. Brilli sottolinea, proprio nell'incipit del suo volume, che «quello che non cessa di sorprendere, nel dibattito anche recente sulla satira, è l'assenza di ogni riferimento all'entroterra storico e critico di questa pratica linguistica e figurativa [...] non ha promosso una riflessione sulla storia, i metodi, le tecniche e le ideologie della rappresentazione satirica», BRILLI, Attilio, op. cit., p. 7.

5. CARNAZZI, Giulio, La satira politica nell'Italia del Novecento, Principato, Milano, 1975, p. 2, cit. in COCCO, Maurizio, «Le vespe qualunquiste e la satira politica», Diacronie. Studi di Storia Contemporanea: La satira fa storia. Eventi, pratiche, linguaggi, 29/10/2012, URL: < http:// www.studistorici.com/2012/10/29/cocco_numero_11/ >, p. 14.

6. CHIESA, Adolfo, La satira politica in Italia, Roma-Bari, Laterza, 1990, p. 6, cit. in COCCO, Maurizio, op. cit., p. 13.

7. FERNÁNDEZ, Iñigo, «Los mexicanos somos... El chiste como autorepresentación y estereotipo del mexicano», Diacronie. Studi di Storia Contemporanea: La satira fa storia. Eventi, pratiche, linguaggi, 29/10/2012, URL:< http://www.studistorici.com/2012/10/29/fernandez_numero_11/ >, passim.

8. EVANGELISTI, Francescomaria, GUTIÉRREZ JIMÉNEZ, Maria Eugenia, «La satira come prodotto culturale: La letteratura di cordel nella Spagna della prima metà dell'Ottocento», Diacronie. Studi di Storia Contemporanea: La satira fa storia. Eventi, pratiche, linguaggi, 29/10/2012, URL: < http:// www.studistorici.com/2012/10/29/evangelisti-gutierrez-jimenez_numero_11/ >, passim.

9. BALESTRA, Luigi, op. cit., p. 2.

10. Ibidem.

11. COCCO, Maurizio, op. cit., passim.

12. ZANDONÀ, Valerio, «La satira: uno specchio dell'antipolitica nell'Italia giolittiana», Diacronie. Studi di Storia Contemporanea: La satira fa storia. Eventi, pratiche, linguaggi, 29/10/2012, URL: < http:// www.studistorici.com/2012/10/29/zandona_numero_11/ >, passim.

13. De POLI, Barbara, «Dal dissenso alla rivoluzione: satira e potere nel mondo arabo contemporaneo», Diacronie. Studi di Storia Contemporanea: La satira fa storia. Eventi, pratiche, linguaggi, 29/10/2012, URL: < http://www.studistorici.com/2012/10/29/depoli_numero_11/ >, p. 22. La citazione di Bergson è tratta da BERGSON, Henry, Il riso. Saggio sul significato del comico, Roma-Bari, Laterza, 2009, p. 28.

14. DE POLI, Barbara, op. cit., p. 1.

15. De TORO MUÑOZ, Francisco Miguel, «La sátira y el humor político durante el Tercer Reich. Los delitos contra la Volksgemeinschaft», Diacronie. Studi di Storia Contemporanea: La satira fa storia. 
Eventi, pratiche, linguaggi, 29/10/2012, URL: < http://www.studistorici.com/2012/10/29/detoro_numero_11/ >.

16. DE POLI, Barbara, op. cit., p. 2.

17. BRILLI, Attilio, op. cit., p. 12.

18. COCCO, Maurizio, op. cit., p.2.

19. VISCARDI, Marco, «Un'altra visione delle cose. Note sulla satira nel Risorgimento», Diacronie. Studi di Storia Contemporanea: La satira fa storia. Eventi, pratiche, linguaggi, 29/10/2012, URL: < http:// www.studistorici.com/2012/10/29/viscardi_numero_11/ >, passim.

20. FERNANDEZ, Iñigo,op. cit., passim.

21. MORELL, Constantino Gonzalo, «Los barrios ríen de la ciudad. Las acciones satíricas del movimiento vecinal español. El caso de Valladolid, 1970-1986», Diacronie. Studi di Storia Contemporanea: La satira fa storia. Eventi, pratiche, linguaggi, 29/10/2012, URL: < http:// www.studistorici.com/2012/10/29/morell_numero_11/ >.

22. EVANGELISTI, Francescomaria, GUTIÉRREZ JIMÉNEZ, Maria Eugenia, op. cit., p. 3.

23. FERNANDEZ, Iñigo,op. cit., passim.

24. PASQUINI, Dario, «La satira e la storia delle emozioni. Una relazione privilegiata?», Diacronie. Studi di Storia Contemporanea: La satira fa storia. Eventi, pratiche, linguaggi, 29/10/2012, URL: < http:// www.studistorici.com/2012/10/29/pasquini_numero_11/ >.

25. EVANGELISTI, Francescomaria, GUTIÉRREZ JIMÉNEZ, Maria Eugenia, op. cit., p. 17.

26. BRILLI, Attilio, op. cit., p. 7-8

27. COCCO, Maurizio, op. cit., p. 13.

28. VISCARDI, Marco, op. cit., p. 4.

29. EVANGELISTI, Francescomaria, GUTIÉRREZ JIMÉNEZ, Maria Eugenia, op. cit., p. 2.

30. DE POLI, Barbara, op. cit., p. 4.

\section{AUTORI}

\section{ALESSANDRO CATTUNAR}

Alessandro Cattunar è Dottore di ricerca in Storia Contemporanea; ha conseguito il titolo presso Istituto italiano di Scienze Umane - Sum (Firenze, Napoli) sviluppando una ricerca dal titolo Il confine delle memorie. Evoluzione delle memorie e processi identitari tra politiche pubbliche e percorsi privati nel goriziano: 1943-1955 sotto la tutela di Marta Verginella. Ha conseguito la Laurea specialistica in Storia d'Europa presso l'Università di Bologna con una tesi dal titolo Il ricordo goriziano. Identità di confine e memorie divise: 1943-1947 - Il confine orientale d'Italia tra documenti e racconti di vita (rel. Mariuccia Salvati). È laureato anche in Discipline del cinema (Bachelor's degree) e ha conseguito un titolo di perfezionamento in «Media education: media, storia, cittadinanza» presso l'Università Cattolica di Milano. Le sue ricerche si focalizzano sulle problematiche della memoria e dell'identità nelle zone di confine, sull'analisi delle fonti orali e sul rapporto tra ricerca storica e media. Su questi argomenti sono in corso di pubblicazione saggi sulle riviste "Italia contemporanea" e "Storicamente". Attualmente con l'Associazione Quarantasettezeroquattro (www.quarantasettezeroquattro.it) sta sviluppando a Gorizia un progetto dal titolo Topografia della Memoria - Museo Diffuso della Città. Percorsi multimediali e interattivi tra i luoghi della memoria e la memoria dei luoghi (www.stradedellamemoria.it). È membro 
dell'Associazione italiana di Storia Orale (AISO) e responsabile del suo sito, www.aisoitalia.it. Dal 2008 collabora con l'Istituto Friulano per la Storia del Movimento di Liberazione (IFSML) di Udine dove si occupa dell'archivio fotografico, e con l'Istituto storico di Torino (ISTORETO) dove si occupa di Media Education. Fa inoltre parte del comitato scientifico per la realizzazione della mostra multimediale I giovani e la Costituzione. Nel 2007 ha collaborato con il settore audiovisivi dell'Istituto storico Parri di Bologna occupandosi dell'utilizzo e della catalogazione delle videointerviste. Dal 2003 si occupa di produzioni audiovisive (regia, produzione esecutiva) e tiene attività didattica approfondendo i rapporti tra tecniche cinematografiche, storia e letteratura. URL: < http://www.studistorici.com/2008/09/14/alessandro-cattunar/ >

\section{ALICE DE RENSIS}

Alice de Rensis (Bolzano) ha conseguito nel 2006 la Laurea in Scienze Storiche (curriculum Diritti, Istituzioni e Culture Politiche) presso la facoltà di Lettere e Filosofia dell'Università di Bologna con una tesi di ricerca sul carteggio inedito tra lo storico ed economista J. C. L. Simonde de Sismondi e Julia Garnett Pertz (1826-1829), trascritto in edizione critica sotto la direzione della Prof.ssa Francesca Sofia, e presentato in occasione della X. Giornata annuale di Studi Sismondiani (2007).

Iscritta alla Laurea Specialistica in Storia d'Europa presso il medesimo ateneo, ha partecipato nell'a.a. 2007/08 al progetto “Cursus Intégré Franco-Italien d'Histoire Européenne Comparée" che, in collaborazione con l'Université Paris VII Denis Diderot, è volto al conseguimento di un doppio diploma, italiano e francese.

Si è laureata nel marzo 2009, sotto la direzione delle Prof.sse Francesca Sofia e Gabrielle Houbre, con una tesi dal titolo Salotti e scritture di courtisanes nella Parigi della seconda metà dell'Ottocento. Le sue ricerche si rivolgono, in generale, allo studio della sociabilità in rapporto a problematiche di genere nel XIX secolo e alle pratiche di scrittura femminili, in particolare carteggi e memorie, nel loro porsi a metà strada tra pubblico e privato.

Ammessa al perfezionalmento presso la Scuola Normale Superiore di Pisa, attualmente lavora come mediatrice culturale presso il "Museo Archeologico dell'Alto Adige" e collabora col "Frauenmuseum-Museo delle Donne" di Merano. È recentemente entrata a far parte delle redazione della rivista on-line "Fillide" (www.fillide.it) ed è membro esterno della Commissione Pari Opportunità del Comune di Bolzano.

URL: < http://www.studistorici.com/2008/09/14/alice-de-rensis/ > 